\title{
Liquid Cell Electron Tomography for Biomedical Applications
}

William J. Dearnaley ${ }^{1}$, Beatrice Schleupner ${ }^{1}$, Nick A. Alden ${ }^{1}$, Floricel Gonzalez ${ }^{2}$, Birgit Scharf ${ }^{2}$, Madeline J. Dukes ${ }^{3}$, and Deborah F. Kelly ${ }^{1,2}$

1. Virginia Tech Carilion Research Institute, Virginia Tech, Roanoke, VA, USA

2. Department of Biological Sciences, Virginia Tech, Blacksburg, VA, USA

3. Applications Science, Protochips Inc., Raleigh, NC, USA

Transmission Electron Microscopes (TEMs) are ideal tools to visualize the intricate details of molecular entities at atomic resolution. To survive the high vacuum system of a TEM, samples are typically embedded in thin films of vitreous ice, sugars, resins, or heavy metal salts. Naturally, these procedures yield only static snapshots of dynamic processes. Recent advances in the synthesis of nanometer-thin materials such as silicon nitride ( $\mathrm{SiN})$ and graphene provide new substrates to serve as controlled liquid chambers for high-resolution imaging $[1,2]$. In combination with specifically designed microfluidic specimen holders (Fig.1A), macromolecules can now be maintained in solution while viewed in a TEM. This technical breakthrough enables us to see progressive events, for the first time, at the atomic level [3]. We refer to this innovation as Liquid Cell-Electron Microscopy (LC-EM) as experiments can now be performed in a native liquid environment within the EM column. The use of LC-EM permits us to peer into the dynamic world of biological macromolecules at unprecedented spatial and temporal resolution.

To extend the current application of LC-EM to study biological objects in 3D, we are developing electron tomography applications, similar to current methods in the cryo-EM field. As a model system, we examined bacteria cells and their natural viral pathogens, bacteriophages (Figure 1B, C). In preparation for EM imaging experiments, equal volumes of cells and purified phage suspension were added to a microfluidic chamber and incubated for 10 minutes. The liquid-enclosed specimen was inserted into a FEI Spirit BioTwin TEM operating at $120 \mathrm{kV}$. Images were acquired at 15,000x magnification at a defocus value of -1.5 microns using a FEI Eagle CCD camera. Images revealed flagellated bacterial cells and phage particles in the liquid cell environment (Fig. 2). The concentration of phage particles was highest near the flagella filaments, signifying the first step of phage-host interaction. We further observed phage particles proximal to the bacterial cell body, indicative of the second phase of interaction, namely phage docking to the bacterial cell surface. These observations are consistent with the notion of phage infection events (Fig. 2, inset).

To ensure that phage particles were authentically interacting with the bacteria, we tilted the specimen within the microscope column and acquired tomographic information. Example images acquired from a $0^{\circ}$ and $45^{\circ}$-tilted specimen are indicated in Figure $2 \mathrm{~A}, \mathrm{~B}$. Performing tilt analysis in the TEM permitted us to further segment 3D interactions and to eliminate the possibility that phages are located above or below the bacterial plane rather than clearly interacting with the expected surfaces. We also distinguished features in the images based on electron scattering properties. Objects with similar scattering properties indicated similar electron densities within the liquid layer. These properties were quantified based on statistical significance at difference threshold values. Threshold cutoffs were colored to show scattering properties ranging from high to low density in Figure 2C, D. For real-time recordings, raw images and colored versions of the images based on electron scattering can be used to show changes in phage movements and attachment properties. 
References:

[1] Dukes, M. J., et al, Chem. Comm. 49 (2013), p. 3007-3009.

[2] Ring, E. A., et al. J. Microsc. 243 (2011), p. 273-283.

[3] Yuk, J. M. et al. Science 336 (2012), p. 61-64.
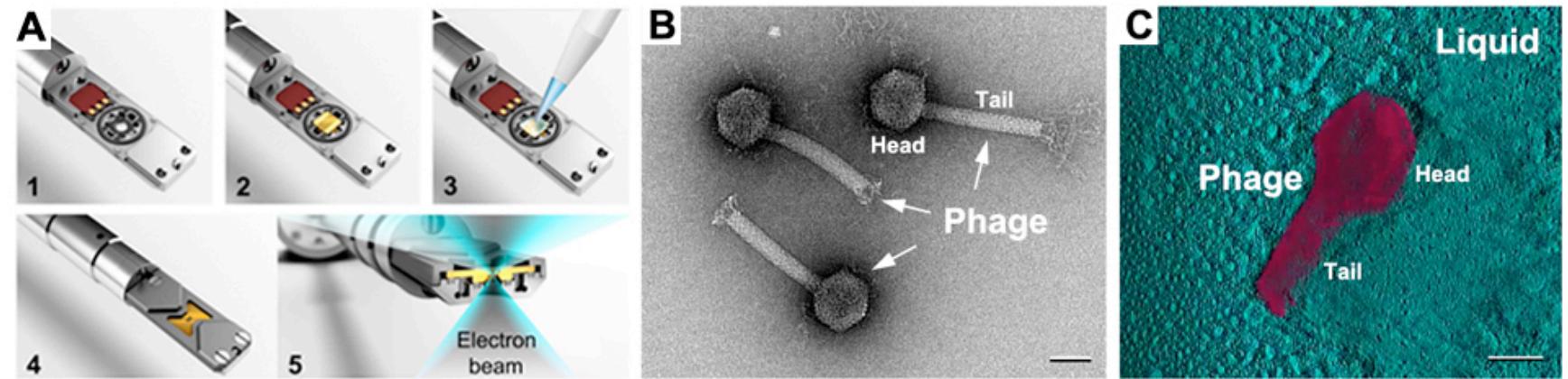

Figure 1. Liquid Cell Electron Tomography of bacteriophage. (A) The Poseidon holder can be loaded with liquid (steps 1-4) for EM imaging. Purified phage particles were assessed in negatively stained specimen (B) and in liquid (C). Phage head and tail features are indicated. Scale bars are $50 \mathrm{~nm}$.
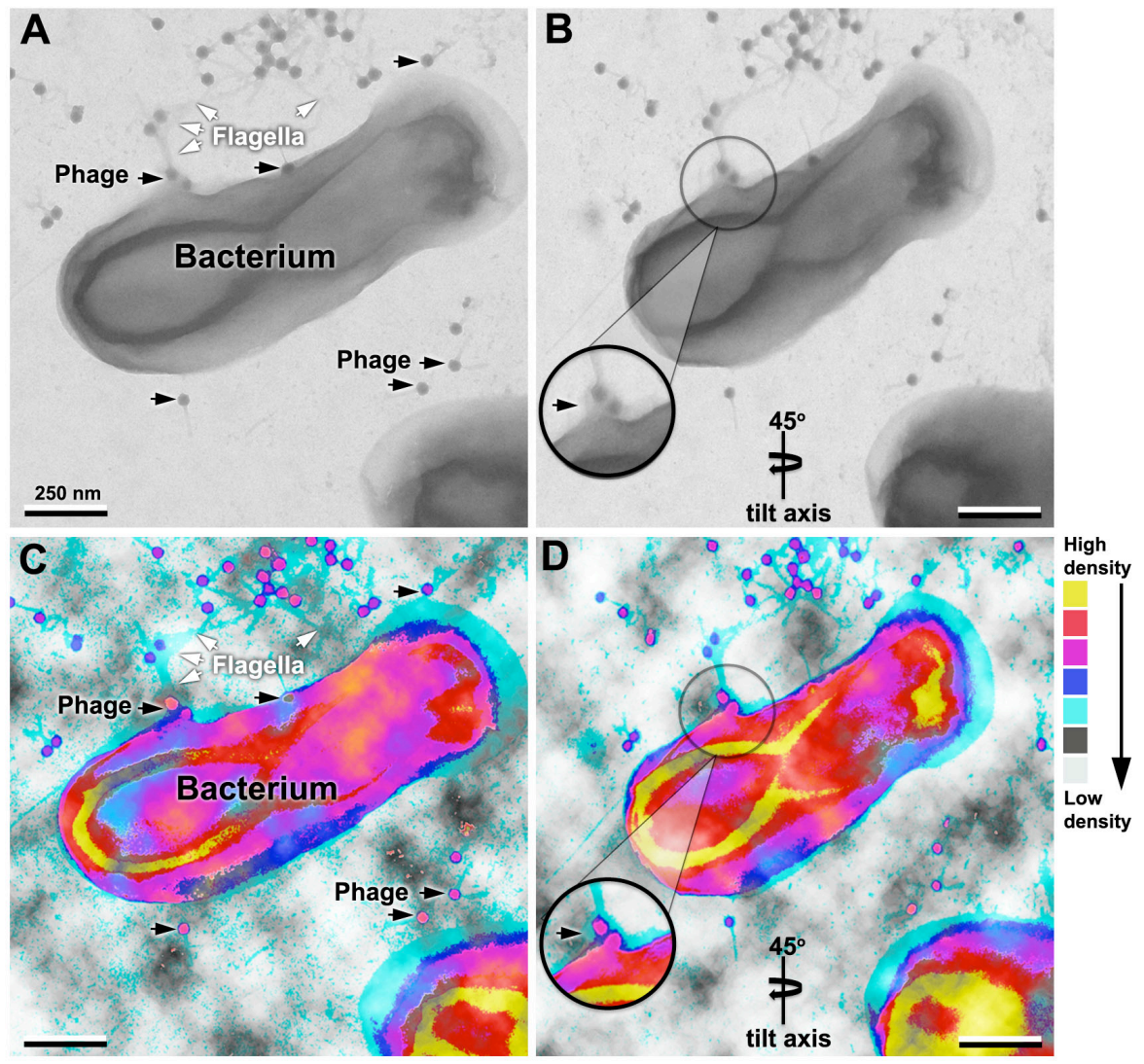

Figure 2. Liquid Cell EM images and processing of phage-bacteria interactions. (A) Images of a bacteria and phage mixture at $0^{\circ}$ and $45^{\circ}$-tilt (B). Black and white arrows point to phages and bacterial flagella, respectively. Colored images show electron density of objects in the untilted (C) and tilted (D) specimen. Inset shows phage heads (magenta) in contact with cell body (cyan). Colors range from high to low density based on threshold measures of electron scattering properties. Scale bar are $250 \mathrm{~nm}$. 